\title{
La gestión del aprendizaje Algunas preguntas y respuestas sobre en relación con el desarrollo del pensamiento en los estudiantes
}

\section{Santos Soubal Caballero*}

\begin{abstract}
Resumen: La gestión del aprendizaje es una obra que refleja el quehacer educacional de los educadores que tienen la responsabilidad de formar al ser humano en la sociedad del conocimiento, bajo la óptica de la educación crítireflexiva que permita al humano insertarse en la sociedad eficientemente. La condición cambiante del mundo contemporáneo hace que el concepto de aprendizaje tome una dimensión más amplia y que se maneje en función del cambio en el significado de la experiencia, para que puedan desarrollarse los humanos con comportamientos a la altura de los tiempos en que vivimos y no a los anteriores, en que el aprendizaje era el símbolo del que más sabía. El aprendizaje es hoy algo que esta en estrecha vinculación con la formación cognitiva, afectiva, valórica y motriz, a partir de la visión holística que se requiere para poder mirar los fenómenos desde una óptica más global que nos permita ver el proceso ante nosotros como una complejidad justo en la medida de lo que es. La gestión del aprendizaje es lo que se requiere para lograr estos propósitos.
\end{abstract}

Palabras clave: aprendizaje, gestión, formación humana, holisticidad, sociedad del conocimiento, formación cognitiva, afectiva, valórica y motricia.

\section{The learning process management. Some questions and answers in regards the development of thinking in students}

Abstract: The management of learning is a work which reflects the educational task of educators that have the responsibility to form to the human being in the society of knowledge, under the viewpoint of the critic-reflexive education that will permit human beings to be inserted efficiently in society. The changing condition of contemporary world gives the concept of learning a more extensive dimension, and demands that it be handled in function of the changes in the meaning of experience, so humans can develop up to the behaviors of these time in which we live and not up to the previous, in which learning was the symbol of the one who knew more. Learning is today something which is in close connection with cognitive and emotional formation, as well as on values and motricity, springing from the holistic vision which is required to see phenomena with a wither perspective that will allow us to see the process that opens before us as a complexity which can be measured exactly for what it is. The management of the process of learning is what is required in order to achieve these purposes.

Key words: learning, management, human formation, holisticity, society of knowledge, emotional, cognitive formation, on values and on motricity.

Recibido: 21.08 .08

Aceptado: 08.10 .08

$* * *$

* Universidad Bolivarana, Santiago, Chile. Email: ssoubal@gmail.com 


\section{El desafío en la educación contemporánea}

Aprender de las estructuras de la vida como punto de partida universal para entender qué es el aprendizaje.

Dr. Osvaldo Panza Doliani

En el nuevo siglo se enfrentan enormes desafíos producto de la evolución de las sociedades modernas cada vez más interrelacionadas universalmente. La relación educación-sociedad ha sido un diálogo permanente a lo largo del desarrollo histórico-social, así lo demuestra las diferentes etapas por la que han pasado las sociedades. La esclavitud en la que el hombre era dueño del hombre, la edad media en la que el hombre era dueño de la tierra, la edad moderna en la que el hombre era dueño de los medios de producción; ¿y en la sociedad posmoderna?, ¿De qué será dueño el hombre?

Por ello la sociedad pos moderna va adquiriendo una importancia significativa, puesto que le está demandando a la educación a partir del diálogo permanente que establecen, una formación humana en el seno de las instituciones escolares que deben responder a las necesidades de la sociedad en curso. La gestión educacional debe enfilar sus acciones principalmente en el sentido de insertar en la sociedad a los individuos de forma eficiente para que cada actividad que desarrollen, cualquiera sea, logre los mejores resultados con el mínimo de recursos posibles.

Un modelo educacional como el que hasta el momento persiste enfilado hacia el saber, con las concepciones del profesor como centro, la enseñanza en el foco central del proceso, una dirección predominantemente autoritaria con curriculum rígidos y comunicación unidireccional del profesor al estudiante, la reproducción memorística de lo que dice el profesor, es excluyente y no posibilita la inserción de ese humano en la sociedad. Sus capacidades intelectuales están pobremente desarrolladas, y por lo tanto estas siempre estarán en el rango de lo potencial y no lo real.

Por lo tanto con el advenimiento de la Sociedad del Conocimiento como algunos le llaman o la sociedad pos moderna como le denominan otros, en que se ha experimentado un crecimiento abrumador del conocimiento debido a la modernización y la globalización en que se ha visto envuelto el mundo de hoy, necesita un humano distinto que sea el resultado de un proceso educacional centrado en el estudiante, en el desarrollo de sus capacidades y valores, que sepa hacer, que aprenda en red, que se inserte en una dirección participativa con curriculum flexible y comunicación bidireccional dirigida al desarrollo de la inteligencia como meta capacidad e integración de múltiples procesos que se dan en las dimensiones cognitivas, afectivas, valóricas y motrices. O sea, que sus capacidades creativas estén trabajadas para lograr lo real y no lo potencial.

Este asunto de singular importancia va requiriendo cambios sustanciales en el proceso docente-educativo o mas específicamente en el proceso del aprendizaje en las instituciones 
escolares. Para la gestión de estos nuevos alcances en el contexto educacional por parte de los gestores educacionales, se requiere concebir y dirigir las influencias en el sentido siguiente:

Primero; En la manera que propiciemos el desarrollo mental de los humanos a partir de la estructura y dinámica de la vida con vista a comprenderla después.

Segundo, en la manera que el sujeto construye conocimientos y aprendizajes a partir de su propio auto desarrollo mental en correspondencia con la auto-organización y desarrollo de los seres vivos.

Tercero, en la comprensión por los humanos de los procesos que operan en sí mismo, con el fin de adquirir significado en la experiencia cotidiana y saberla auto valorar y que ulteriormente las acciones sean conscientemente realizadas.

Por supuesto, toda esta gestión que se requiere hace reflexionar sobre la naturaleza compleja de la problemática. Es necesario pues referirnos en cierto modo a procesos desarrolladores a escala de la mente humana como en cierta forma se desarrolla la vida. Por eso se escogió la estructura de la vida su génesis y evolución, para que sirva de referencia en el enfoque metodológico a tratar y que resulta muy conveniente cuando tratamos a los humanos que aprenden en el contexto en el que se desarrollan.

La vida que es un dilema cuajada de contradicciones y de emergencias requiere en primera instancia que todo ser vivo se comprenda él y el mundo que le rodea para poder seguir vivo y actuar, es decir, transitar en la dinámica intrínseca del sistema organismo /entorno; Una estructura capaz de adaptarse al cambio, convivir con él, y proyectar nuevas transformaciones.

Al respecto Humberto Maturana plantea:" "La formación humana tiene que ver con el desarrollo del niño o niña como persona capaz de ser co creadora con otros de un espacio humano de convivencia social respetable" y, continua diciendo: "Por eso la formación humana como tarea educacional, consiste en la creación de condiciones que guían y apoyan al niño o niña en su crecimiento como un ser capaz de vivir en el auto respeto y respeto por el otro..."

Ese cambio que se empieza a vislumbrar desde la segunda mitad del siglo XX y que fue un duro golpe al positivismo, es motivado entre otras cosas por el cúmulo de conocimientos que se generaron en medio de una revolución científico-técnica de gran alcance, que ha provocado el cambió de una sociedad estática a una cuajada de interrelaciones, emergencias, con el tratamiento a las subjetividades humanas como centro,

${ }^{1}$ Maturana H. (1995) Proposición para la Educación. S/E. República de Chile 
y que no se debe ver sino es en su justa medida a partir de lo complejo. Esta complejidad social que cada vez más se define requiere de un enfoque como el complejo, que transita por las interrelaciones, que encara el desorden, la confusión y la incertidumbre. Muchos por desconocimiento la utilizan como palabra problema y no la enfocan desde el punto de vista de la palabra solución. Como complemento auxiliador, la complejidad no evita el desafío sino lo enfrenta y lo resuelve.

Esta forma de entender la realidad se ha ido posicionando en todos los saberes, en la física, la biología, así como en las ciencias del comportamiento humano y las sociales entre otras. Cada vez más se suceden los descubrimientos como avalancha incontenible y, a su vez, son mayores los modelos teóricos que se construyen para tratar de dar explicación a la realidad circundante.

Esta forma de interacción social contribuye a que el ser humano evolucione y pueda desarrollar su mente como un proceso de auto desarrollo, y en la medida que lo logre tendrá una capacidad mayor para poder comprender el mundo y sobre todo transformarlo.

Al respecto el Dr. Panza Doliani plantea: "Aprender de las estructuras de la vida como punto de partida universal para entender qué es el aprendizaje".

Sin embargo, la educación transita por un camino que no vislumbra respuesta al modelo social que se esta imponiendo hoy en día, entre otras cosas por la influencia marcada del positivismo en lo filosófico, el conductismo en lo psicológico, y los modelos tradicionales pedagógicos que aun están fuertemente enraizados en el pensamiento de los docentes. En lo fundamental, la educación ha perdido su propia identidad, hoy más acentuada, ya que se prepara a los humanos para servir en una modalidad específica de las estructuras económicas, políticas y sociales, sin concebir en muchos casos como premisa la educación de la comprensión humana para insertarse después en estas estructuras.

No se prepara al estudiante futuro profesional para insertarse en la sociedad como un ser auto referente, creativo, reflexivo, que se evalúa a sí mismo en su crecimiento, decrecimiento y errores, y no desarrolla un estilo crítico como producto de la educación crítica recibida en la institución escolar. Enseñar no es tan sólo una cuestión de conocimientos, sino de modos de razonar.

El predominio del pensamiento reproductivo en los niveles escolares y el tratamiento reduccionista y analítico que se da al curriculum, impide que se logren los propósitos de

\footnotetext{
${ }^{2}$ Panza Dolíani O (2005) Apuntes para una reflexión sobre las estructuras de la vida y su relación con el aprendizaje. Universidad Tecnológica de Chile. Conferencia Magistral dictada el 6 de octubre 2006 en él anfiteatro de la institución. La Serena. Chile
} 
una educación como sistema complejo.

Convertir, transformar o cambiar las concepciones hacia una nueva educación en la gestión de directivos y docentes serán los desafíos que la educación tendrá que enfrentar en el siglo XXI.

\title{
2. Análisis actual de las dificultades que provoca una insuficiente educación del pensamiento
}

\author{
Toda naturaleza compleja no es acumulativa, y \\ cualquier suceso puede cambiar el curso de la historia. \\ Dr. Fernando González Rey
}

Dentro de los desafíos a enfrentar por la educación en el actual siglo se encuentra la educación del pensamiento a partir de un enfoque que acerque la pedagogía a la evolución de la vida. Lo anterior presupone un análisis más integrador de fenómenos como subjetividad, cultura, desarrollo, aprendizaje, sistemas, entre otros, que nos posibilita tener un dominio mayor con vistas a arribar a conclusiones integradoras sobre la relación causa problemas - alternativas de solución, que se producen en el proceso docente educativo y en particular en la estimulación del pensamiento.

La estimulación del pensamiento es un factor que los docentes necesitan desarrollar para lograr mayor eficacia en el graduado que se pretende formar, y su fundamento se encuentra dado en el conocimiento de la estructura y funcionamiento de la vida, a partir de la cual comienzan todos los análisis desde la enseñanza elemental.

Sin embargo, la pobre utilización del pensamiento en sus aristas reflexiva y creadora, repercute en prácticas predominantemente centradas en el desarrollo de los contenidos plasmados en los programas escolares sin una concepción de lo que significa el autodesarrollo en los sistemas y en particular el de organismo/entorno que nos lleva a considerar el conocimiento como una propiedad auto-organizativa del cerebro, del sistema nervioso acoplado al medio ambiente.

Un ejemplo puede ser el siguiente:

Al inicio del curso o semestre el docente se preocupa por pasar el contenido, en la mayoría de las veces sobre dimensionado en horas sin ir a la esencialidad y teniendo el criterio que el alumno debe saberlo todo. No identifica en muchos casos el nivel de los conocimientos previos de los estudiantes por intermedio del diagnóstico, como punto de partida para el proceso. En otros casos se diagnostica, pero se hace mal, puesto que se aplican "pruebas de diagnóstico" al primero o segundo día de clases lo que no involucra al estudiante ni lo hace participe del mismo, lo que provoca que los resultados estén viciados por la falta de motivación, el desinterés, él quiebre con la vida y la pobre interacción con el 
estudiante. Los resultados se quedan como información para la institución sí acaso. En síntesis, no se concibe el punto de partida para el desarrollo propio del estudiante y se sigue considerando el conocimiento como algo que se acumula semejante a un almacén de insumos.

El semestre, estructura artificial del tiempo concebido en la educación, sigue su desarrollo y se convierte en una "pista de carrera" en que los profesores cumplen, pero los alumnos en muchas oportunidades no, porque aún no se concibe el aprendizaje como una modificación cerebral de carácter holístico y, para que sea significativo, necesita de la reconfiguración de todo el cerebro y la corporeidad humana. ${ }^{3}$ Toda naturaleza compleja no es acumulativa y el suceso más insignificante puede cambiar el curso de la historia.

No hay flexibilidad porque los que elaboran los programas de las asignaturas desconocen que la aptitud y flexibilidad cognitiva de los organismos vivos fue adquiriendo forma a partir de complicados procesos de aprendizajes. Nuestra evolución orgánica es una evolución cognitiva en interacción con el medio social y nuestra existencia es la de seres aprendientes. Nuestro cerebro se desarrolló a partir de la interacción del humano con su medio ambiente que aseguró un aprendizaje, y por tanto nuestra evolución conserva las huellas de sus diversas fases como elementos que participan en los procesos cognitivos actuales.

A manera de síntesis, la pobre estimulación del pensamiento por parte de los docentes se circunscribe a:

- El desconocimiento por los docentes de lo que es el pensamiento, su génesis y sus funciones en relación con la estructura y dinámica de la vida.

- El diagnóstico inicial para emprender el proceso de desarrollo del aprendizaje no está sustentado científicamente o no se hace, lo que impide recopilar una información veraz acerca de los conocimientos previos.

- No son consideradas las actividades docentes sistemáticas y dentro de ellas las tareas, como un instrumento de diagnóstico periódico que posibilita la estimulación del pensamiento en los estudiantes.

- El proceso formativo se considera espontáneo debido a que se parte de la "cantidad de conocimientos" que almacena el estudiante y todo se supedita a traspasar conocimientos y medirlos, como dicen los docentes y directivos.

- Las tareas son marcadamente reproductivas y la evaluación no es vista como estrategia de aprendizaje sino para "medir" cantidad de conocimientos.

- No se parte de la necesidad como fuerza motriz del desarrollo humano y por tanto el

\footnotetext{
${ }^{3}$ González Rey F (1997) Epistemología analítica y subjetividad. Editorial Pueblo y Educación. La Habana
} 
estudiante al no explicitarla no se motiva.

- El insuficiente dominio teórico-práctico de las características del pensamiento.

\title{
3. Fundamentos psico-pedagógicos y didácticos para el desarrollo del pensamiento de los estudiantes
}

\author{
Pensador es aquel que cultiva la dinámica de su propio pensamiento, que \\ estará vivo si reconoce los propios límites de su lenguaje. \\ H. Assman
}

Para lograr un pensamiento activo en los estudiantes se precisa desarrollar sus etapas superiores, como son: Análisis, síntesis, comparación, abstracción y generalización, puesto que el estudiante se supone tiene formadas sus estructuras cognitivas referidas al pensamiento lógico y se debe tratar el pensamiento lateral y consolidar el desarrollo de ambos. Intervienen en él múltiples alternativas que los docentes no deben descartar, puesto que las insuficiencias de una para explicar un tema puede ser complementado por otra que la aborda desde otra perspectiva.

Recordar que, ${ }^{4}$ pensador es aquel que cultiva la dinámica de su propio pensamiento que estará vivo si reconoce los propios límites de su lenguaje.

Estas ideas concretan el cambio que se debe dar en los directivos y docentes para enfrentar su gestión en la institución y en la sala de clases respectivamente, por lo que deberán promover la capacidad meta cognitiva, o sea, para comprenderse a sí mismo, sus procesos mentales internos para lograr una verdadera concientización de su aprendizaje como elemento inicial para poder comprender los procesos cognitivos, afectivos valóricos y motrices que se dan en los estudiantes a los que apoyamos en el proceso de su propio aprendizaje. ¿Cómo se puede entender algo sino somos capaces de experimentarlo nosotros? Deberá ayudar a los estudiantes como aprendientes a, aceptar, celebrar y entender el vínculo del lenguaje con los procesos cognitivos neuronales.

Por supuesto, esto requiere del perfeccionamiento de directivos y docentes para esta compleja tarea centrada en la metodología para el desarrollo de capacidades más que prepararlos en contenidos, para la búsqueda de soluciones cualitativas permeadas de flexibilidad e integración dentro del proceso docente educativo.

El desarrollo de la mente de los estudiantes, requiere de una reformulación de la

\footnotetext{
${ }^{4}$ Citado por Assman H (2002) en Placer y ternura de la educación. "Hacia una sociedad aprendiente". Ediciones Narcea SA Madrid. España
} 
gestión docente cuyas bases se dan a continuación:

- El entendimiento de la educación como un proceso que propicia y desencadena la autoorganización de la mente humana y el lenguaje de las personas.

- Disminución de la simplicidad y paso a la complejidad, de manera tal que, el pensamiento integre los modos simplificadores del pensar.

- El desarrollo del pensamiento sistémico que presenta la multidisciplinaridad o multidimensionalidad como condición para el desarrollo de la mente humana y comprenda en un fenómeno, la distinción y a la vez la unión.

- Quien enseña sólo ha de mostrar elementos que sirvan para la comprensión y propiciar el ritmo del lenguaje.

La aplicación consciente de una metodología de gestión teniendo en cuenta lo señalado con anterioridad, permite comprender la naturaleza así como la formación y desarrollo del pensamiento humano.

Por ello circunscribirnos al pensar como parte de la evolución del organismo vivo en su interacción con el medio ambiente, puede explicar la interacción que sé da entre el sujeto y el objeto de estudio visto como una unidad, por lo que promueve, ya que une, contextualiza, globaliza y al mismo tiempo reconoce lo singular, lo individual y lo concreto. Desencadena en el ser humano tener en cuenta el texto y el contexto, el ser y el entorno, lo local y lo global, lo multidimensional, en síntesis, lo complejo. La comprensión hacia los demás necesita de la complejidad de la comprensión humana.

Por supuesto, de lo anterior se desprende que, el desarrollo del pensamiento sólo es posible en la actividad contribuyendo a ello la comunicación que se establece entre los humanos por intermedio del lenguaje. De esto se infiere que, la formación y desarrollo del pensamiento esta condicionado históricamente por intermedio de las interacciones con objetos, fenómenos, acontecimientos y otros humanos, que provoca conceptos, juicios, ideas, modelos teóricos, que se convierten en valores culturales y que a su vez sirven de base a nuevas cualidades que se van observando en nuevos acontecimientos, procesos y fenómenos.

Lógicamente, el pensamiento será entonces el resultado no de una actitud pasiva o contemplativa en la sala de clases, sino de la interacción que se produce entre sujeto y realidad, transformándose él mismo y el mundo que le rodea por intermedio de la cultura. Aquí converge lo personológico y lo mediacional.

Si esto es comprendido vale la pregunta siguiente: ¿Por qué se actúa en la sala de clases de otra forma?

La respuesta a esta pregunta hay que buscarla en las siguientes consideraciones didácticas: 
- Un proceso en que los alumnos tomen decisiones por sí mismo.

- El trabajo en equipos y la integración a una clase activa en la sala.

- El asumir responsabilidades.

- El poseer estructuras cognitivas formadas en la etapa del pensamiento lógico.

- El afecto que requiere el ser humano implícito en sus acciones.

- La presentación de una estabilidad en los procesos psíquicos y biológicos.

\title{
4. El papel de la tarea escolar en el desarrollo del pensamiento de los estudiantes
}

\author{
"La tarea es el portador natural del auto desarrollo del \\ pensamiento estudiantil" \\ Dr. Santos Soubal
}

Juega un papel importante y significativo en la gestión del pensamiento en los estudiantes la tarea escolar, no vista a través de un prisma de la simplicidad, sino en el justo medio de la complejidad que la caracteriza.

Es de suponer entonces que, las tareas deben presentar niveles de dificultad mayor apoyados en la re-conceptualización de los conceptos vistos en los niveles precedentes, los nuevos conceptos presentados, así como los modelos teóricos que se presentan junto a la operacionalización de los procesos con vistas a la solución de problemas sean reales o ficticios. Sólo la interacción sujeto-objeto de estudio con lo que arrastra en el sentido cognitivo, afectivo y los valores garantiza la formación y desarrollo de los procesos mentales.

Se ha configurado como regularidad en los docentes el fenómeno de la evaluación como un fin en sí mismo y no como un medio, que posibilita la autovaloración, la reflexión en la adopción de estrategias, el apoyo a otros, entre otras cosas a partir de buenas tareas.

¿Cómo entonces podemos potenciar el pensamiento de los estudiantes a partir de las estrategias de aprendizaje contenida en la tarea escolar que presenta el docente en su gestión?

Se requiere pues por el docente lo siguiente:

-Resaltar la significación del objeto de estudio.

- Presentar el objeto de estudio a partir de una visión holística.

-El enfoque de la complejidad para conducir el ritmo del aprendizaje de los estudiantes en el tratamiento de los temas contenidos en el plan de estudios.

-La operatividad para la aplicación práctica. 
-El enfoque teórico para explicar las relaciones internas de los objetos de estudio.

Por lo tanto podemos inferir que, la significación que hay que darle a las tareas se corresponde con una buena concepción de ellas y no para salir del paso. Las tareas se convierten en una necesidad para docentes y estudiantes vistas de distintos planos de análisis. En el plano estudiantil es una oportunidad de aprendizaje y, en el del profesor, una estrategia de aprendizaje que entrega a sus estudiantes.

En varias conferencias sobre gestión del aprendizaje he planteado: "La tarea es el portador natural del auto desarrollo del pensamiento estudiantil”.

Muchos autores a lo largo de los años han clasificado las tareas escolares a partir de diferentes referencias aunque todos coinciden en afirmar que es la esencia del proceso docente-educativo y que posee una triple finalidad: diagnóstica, formativa y predictivaretroalimentadora.

La triple finalidad se sustenta en los siguientes principios:

- El carácter sistémico.

- La sistematicidad en su aplicación.

- La heterogeneidad de las respuestas que se esperan.

- Comprensión del proceso del aprendizaje del estudiante.

- Carácter transformador que ayuda en el cambio del significado de la experiencia.

Es lógico pensar a partir de los principios establecidos que, la tarea no posibilita que el docente improvise puesto que debe ser meditada y concebida en sistema conjuntamente con el programa, desarrollo de las unidades didácticas y la clase del profesor, de conjunto con las formas en que el docente organice su proceso, dando paso a la reflexión y a la creatividad. Por ello, a las tareas hay que darle el tiempo suficiente en el programa para realizarlas.

Las investigaciones, los proyectos, los estudios de casos, las situaciones problematizadoras, los seminarios, las disertaciones orales, entre otras, como parte de la tarea, son excelentes actividades que propician el desarrollo del pensamiento y sobre todo la constante renovación de métodos, estilos y estrategias docentes para hacer más cualitativo el aprendizaje y que el alumno no se enajene con las nuevas tecnologías de la información, sino que sea capaz de valorar por sí mismo el dominio de sus destrezas y habilidades que se requieren para transitar por la vida como un ser del que forma parte y que ayuda a construirla; que se percaten del sentido subjetivo y significativo del

\footnotetext{
5 Soubal S (2006) Conferencia sobre Gestión del Aprendizaje, Diplomado impartido en la Universidad Tecnológica de Chile. La Serena. República de Chile.
} 
aprendizaje y la ponderación que merece el aprendizaje personalizado construido a partir de la red o puntos que hoy existen que posibilitan un entramado o madeja de alternativas que sabiéndolas utilizar posibilitan aprendizajes verdaderamente significativos.

El insuficiente dominio de las nuevas concepciones didácticas para la Educación Contemporánea para lograr la estimulación y activación del pensamiento en base a un modelo didáctico de carácter teórico, es la causa de las prácticas espontáneas e improvisadas que hoy se detectan en la educación.

Este modelo por excelencia para la educación es el Proceder Investigativo, debido al potencial desarrollador que lleva en sí, que posibilita desarrollar de forma holística muchas de las familias de habilidades que tienen implícitas acciones, como la apertura mental, la indagación y el razonamiento que tributan al desarrollo de las capacidades con vistas a la formación de las competencias genéricas de desarrollo humano imprescindibles para transitar por la vida. El profesor deberá trabajarlas como parte de la gestión del aprendizaje.

\title{
5. ¿Qué es la gestión del aprendizaje en el quehacer educacional de la gestión escolar?
}

\author{
¿Es el aprendizaje basado en la ciencia un motivo \\ obligado de preocupación de los docentes?
}

Se ha definido la gestión escolar ${ }^{6}$ como, el conjunto de acciones que se conciben conscientemente y se ejecutan con vistas al cumplimiento de objetivos que dicen relación con la formación integral de las personas para que puedan insertarse en la sociedad eficientemente. Integrar sus aristas administrativas gerenciales, el quehacer educacional y los valores resulta ser significativo en la gestión y la razón de ser en las instituciones escolares a cualquier nivel.

Esta gestión en las instituciones escolares encaminada específicamente a la conformación y re configuración constante del cerebro de los estudiantes por intermedio de la interacción social con vistas a lograr cambios en el significado de la experiencia en los sujetos, le llamamos gestión del aprendizaje.

Pero, ¿estamos preparados desde el punto de vista de nuestras ideas para enfrentar los cambios en la gestión del aprendizaje que se predicen para el siglo XXI cuando ya estamos adentrados en la sociedad del conocimiento?

\footnotetext{
${ }^{6}$ Soubal S (2004) La gestión escolar. Monografía para el diplomado en gestión del aprendizaje. INACAP- La Serena. Chile
} 
No cabe la menor duda que los cambios paradigmáticos en la ciencia a lo largo del desarrollo social han traído para la mayoría felicidad, para algunos, tristeza, y para otros indiferencias. Sin embargo, la evolución sigue y en muchas ocasiones los que no han intentado insertarse en las transformaciones conscientemente y han seguido actuando en su proceder específico en correspondencia con su experiencia, los resultados en su labor a lo largo de años han sido medianamente eficaces o pobres.

La educación no esta exenta de esa visión. A lo largo de años hemos transitado por paradigmas que respondieron a una época, a un tipo de sociedad y que fueron eficaces en aquel período, pero en la medida que la sociedad evoluciona hacia nuevos niveles cualitativamente superiores múltiples problemas se presentan y hay que estar al tanto de ellos para que a través de nuevos paradigmas educacionales, se solucionen.

En el siglo XIX el paradigma clásico predominantemente autoritario respondía a las exigencias del momento y del contexto, en la primera mitad del siglo XX el paradigma conductual imperaba en la educación y fue quedándose atrás, y por lo tanto nació el paradigma cognitivista que resolvía los problemas referidos a la formación cognitiva de los estudiantes en el contexto del impacto que causó el adelanto científico técnico de la época y las insuficiencias que presentaban los paradigmas anteriores para fundamentar el aprendizaje de los estudiantes.

Estos cambios generaron nuevas tendencias y alteraron las existentes. Los alumnos son otros, el entorno es otro y la sociedad en que estamos es otra más globalizada y modernizada, con la riqueza material como eje central, con el insuficiente tratamiento de lo afectivo y valores. Fíjese cuantas cosas por solucionar tenemos en el cajón de los problemas no resueltos, y aun a pesar de ello existen directivos y docentes en las instituciones educacionales que no interpretan esos cambios y centran su actividad pedagógica en lo cognitivo, sin abordar lo afectivo y valórico como un eco-sistema entre los tres componentes.

El alumno a formar deberá recibir las influencias de otros modos educativos, distintos a los existentes, para que interprete la realidad, sea crítico y humanamente sensible, resuelva los problemas y transforme. ¿No estaremos adentrándonos en otro cambio paradigmático en la educación? ¿El paradigma cognitivista resuelve los problemas en toda la extensión del humano o sólo parcialmente?

Pienso como respuesta a esta pregunta que un nuevo paradigma sirve de soporte a la educación contemporánea.

¿Es el aprendizaje en los centros escolares un motivo de preocupación fundamentada en la ciencia o es la cadena de todos los días aplicada sin importar para nada como se construye? ¿Se domina científicamente por los docentes el proceso de construcción del aprendizaje? 
Muchos autores de estos temas convergen en plantear que el aprendizaje se ve como un fenómeno de almacenamiento de conocimientos y científicamente no se domina su construcción.

En las respuestas a esas interrogantes está mi fundamento, coincidente con las de muchos autores, que permite decir que la gestión del aprendizaje debe de sufrir un cambio en el seno de las actividades pedagógicas, por las limitaciones que ha adolecido en la estimulación del pensamiento en los estudiantes y que no esta dando respuesta a las exigencias que la sociedad ha demandado de la educación.

\section{6. ¿Qué se entiende por actividad pedagógica dentro de la gestión escolar?}

La actividad es la forma específicamente humana de relación con el mundo circundante en que se manifiestan las acciones.

Es la forma específicamente humana de relación con el mundo circundante cuyo contenido constituye un cambio o transformación radical en el significado de la experiencia en los sujetos. Es la educación misma con sus protagonistas, como un momento científico idéntico a cualquier otro, como un científico encerrado en el laboratorio, como un científico cuyo laboratorio es la escuela y su entorno, que debe mirar al alumno como sujeto y objeto de la investigación la que hay que predecir, observar en su evolución, cualificar y evaluar a lo largo del proceso docente-educativo que se desarrolla, en estrecha vinculación con la construcción de conocimientos y la apropiación de estos.

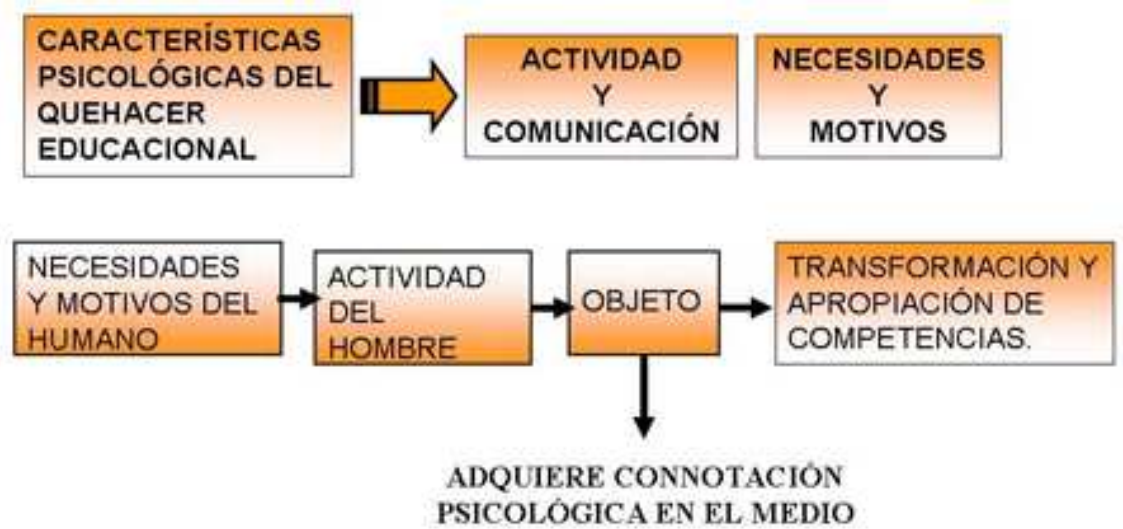

El conflicto que hoy se presenta entre nuestras sociedades y la educación radica en que ésta no satisface plenamente los requerimientos de aquella, entre otras cosas, es el resultado de una brecha que se ha ido manteniendo en la praxis pedagógica a lo largo de muchos años. La educación ha presentado limitaciones en transferir creativamente lo novedoso de las ciencias a la práctica cotidiana de la sala de clases. Lo que se hace es "discursar" y poner en documentos como el Proyecto Educativo Institucional lo que 
teóricamente hay que tratar en la actividad pedagógica del aula, pero no se logra en la realidad concreta. Se ha sostenido por muchos autores que, el cuadro científico del mundo ha sufrido grandes cambios a lo largo del desarrollo de la humanidad, pero en la praxis escolar las ideas clásicas presentadas al comienzo muestran la tendencia a mantenerse en el contenido de diferentes actividades a pesar de algunos intentos por lograr lo contrario.

Existe una tendencia muy marcada en los gestores educacionales hacia la mirada retrospectiva y no a la prospectiva, por lo que se originan reformas cuando debieran existir transformaciones.

Como bien sintetiza Delgado C.: "En los trabajos que se han desarrollado al respecto se distinguen cuatro tipos de revoluciones científicas que pueden identificarse como, Copernicana, Kantiana, cuántico-relativista y científico-técnica. La primera provocó un cambio en la elevación del pensamiento abstracto por sobre el común (es la tierra la que gira alrededor del sol, no siendo lo que se ve), la Kantiana dio un paso hacia él quiebre de la inmutabilidad de la naturaleza, mientras que la cuántica relativista desplazó la evidencia de la mecánica newtoniana y la científico técnica ya en la segunda mitad del siglo XX con el advenimiento de las nuevas tecnologías de la comunicación y el acercamiento del conocimiento a la realidad para transformar, han dejado agónico el cuadro clásico del mundo, para dar paso a uno que se configura sobre una concepción de la realidad como un todo complejo".

\section{¿Cómo la educación se incorpora a este movimiento?}

Esta evolución que ha mostrado el pensamiento universal hasta nuestros días, ha conducido a pensar diferente y es parte de esa nueva forma de pensamiento que se supone debemos asumir en la gestión escolar. No tiene sentido seguir desarrollando actividades pedagógicas para formar un humano para un mundo que ya no existe, pues ello expresaría un desfase con la práctica. Esto provoca que el humano no actúe y se retrotraiga en comportamientos que los excluye del sistema social en términos de enajenación.

\section{7. ¿Qué requiere la educación para ser coherente con lo que necesita la sociedad actual en la gestión de aprendizaje?}

\footnotetext{
"El constructivismo encierra un momento de síntesis de las diferentes escuelas e incluso modelos, pero no como verdades eternas".
}

T.Kuhn

\footnotetext{
${ }^{7}$ Delgado C. Artículo publicado en la revista de Filosofía de las Ciencias Vol 1, 1999 Colombia pág 38 citado por Taeli Gómez aspirante a doctora en ciencias filosóficas en su tesis en opción al diplomado Enfoque Contemporáneo del proceso docente-educativo Chile 2003
} 
El modelo teórico de la gestión del aprendizaje que se debiera aplicar no puede ser un mero capricho de intelectuales, sino debiera ser una consecuencia de una coherente educación - sociedad la que se sostiene sobre un cuadro del mundo que lo ve como una realidad compleja, y el único pensamiento que lo puede comprender es el que se sostiene a partir del enfoque complejo.

Hoy es insostenible que la interpretación de la realidad sea simple, separable, cuantificable, por lo que es imposible entender el proceso del conocimiento y del aprendizaje sobre pilares que estén anclados en esa realidad.

El aula en las que se realizan diversas actividades pedagógicas hay que verla como una realidad que se vive en cada momento, en que los sujetos construyen los conocimientos, es una continuidad de su vida cotidiana, no ideal que se forma para las horas en que el alumno está en la escuela. Esto último es una de las razones entre otras, que profundiza la brecha entre la educación y sociedad.

Para ser coherente con lo que necesita la sociedad contemporánea, la gestión del aprendizaje requiere de actividades pedagógicas que estén sustentadas teóricamente en un modelo en que el alumno interactúe socialmente, modificando sus estructuras cognitivas a partir de sus conocimientos previos en unidad con lo afectivo motivacional y los valores, en un modelo escolar, que simule el contexto social en que se desenvuelve, o sea la vida. El profesor deberá propiciar penetrar en la realidad compleja incluyendo al humano, no como sinónimo de dificultad, sino como alta densidad de interrelaciones que se manifiestan en ella, actuar en ella, entenderla e investigarla conjuntamente con sus alumnos.

La gestión escolar para ser tratada como proceso complejo requiere de las siguientes consideraciones:

- El incremento del número de especialidades.

- El incremento de la virtualidad.

- La integración entre el saber y las ciencias.

- El tratamiento de las aristas afectivas y valóricas.

- El desarrollo de competencias.

- La integración del conocimiento en el producto.

- La creación de redes y trabajo en equipos.

- La obsolescencia del conocimiento rápidamente.

- Los trabajadores del conocimiento son únicos e irrepetibles

- El cambio debe conjugarse con la permanencia humana. La complejidad es dilema.

La visión hacia la complejidad puede contribuir a clarificar el camino hacia un campo que se abre para la educación. Puede provocar además un proceso docente-educativo mejor y más encantador, que posibilite profundizar en las raíces y no transitar por las ramas. Es redimensionar las propias prácticas educativas cuando se tiene el dominio pleno de las 
interrelaciones y se comprende cuál escoger.

Este enfoque da la opción de comprender el aprendizaje en su pleno desarrollo que se logra conducir si en su operacionalización se utilizan procedimientos cualitativos y fundamentalmente los relativos al paradigma investigador cualitativo. Es pues aprender a trabajar la gestión en su dinámica desde una posición en que la bipolaridad como método sea lo que predomine, con una visión holística o integradora cuajada de emergencias que se presentan.

Este enfoque basado en el paradigma de la complejidad, permite comprender el papel del desarrollo humano y su direccionalidad en relación con el aprendizaje. Es recuperar la comprensión de los humanos en su naturaleza interna y evolución, así como sus percepciones de la realidad en un mundo cada vez más complejo.

Para ello los docentes deben comprender que no se trata de seguir impartiendo más y más contenidos en las instituciones escolares, y en particular en las universidades, que pone al docente contra el reloj por su cumplimiento, sino de ir a lo esencial en los conocimientos y habilidades, y darle preferencia al desarrollo de las capacidades cognitivas en un proceso que tenga como base los componentes de la ciencia tales como: valorar la historicidad del contenido, comprender la esencia de los fenómenos en el orden teórico y saberlos aplicar, establecer nexos y relaciones, tener en cuenta los aspectos éticos que acompañan a los descubrimientos científicos y crear un sentido de compromiso social. Por otra parte el vínculo con la vida, para que los estudiantes desarrollen valores que lo comprometan posteriormente a realizar transformaciones en su realidad sin perder lo afectivo.

¿Y qué enfoque se aviene más a las concepciones acerca de la gestión del aprendizaje en relación con la estimulación del pensamiento para interpretarla a partir del enfoque de la complejidad?

La base teórica del enfoque reflejado en un modelo de carácter didáctico se circunscribe a las estructuras de la vida, como un proceso de auto desarrollo que fue adquiriendo forma en la historia a partir de complicados proceso de aprendizaje en el enfrentamiento con la vida misma. Este proceso de búsqueda constante de información para solucionar problemas se torna imprescindible en la educación actual como un sistema adaptativo y complejo y se denomina proceder investigativo, que sujeto a cierta metodología constituye la base de nuestro modelo didáctico.

Este modelo, que integra la complejidad con el proceder investigativo refuerza las tendencias contemporáneas del quehacer educacional hacia el constructivismo.

$\mathrm{Al}$ respecto podemos citar lo siguiente:

"En este modelo se debe conocer que el constructivismo encierra un momento de 
síntesis de las distintas escuelas e incluso modelos, pero no como verdades eternas",8

Clásicos del constructivismo como Jean Piaget entre otros, enfocan sus estudios mas bien a la actividad cognoscitiva y su vínculo con el desarrollo de las estructuras mentales en los humanos más que a las operaciones que el sujeto realiza.

Otro clásico Vygotski, parte de un enfoque dirigido hacia la construcción del conocimiento por medio de operaciones y habilidades cognoscitivas que se indican en la interacción social. Muchos estudiosos de estos temas le llaman el modelo contextual. Introduce este clásico de la psicopedagogía el concepto de zona de desarrollo próximo (ZDP).

El autor de la presente obra dimensionando los aportes de cada uno de estos clásicos, apoyará sus consideraciones en una gestión basada en el enfoque de la complejidad (gestión de la complejidad) y como sustento teórico psicopedagógico, la relación existente entre aprendizaje y desarrollo (gestión del aprendizaje) en un contexto histórico cultural determinado, por considerarlo que se acerca más a los requerimientos de la vinculación escuela-sociedad contemporánea.

\section{8. ¿Cuál entonces es el problema central en la aplicación y puesta en práctica de modelos desarrolladores contextuales dentro de la gestión del aprendizaje?}

En la escuela de hoy el problema central obedece a la no - solución del dilema entre el desarrollo de las potencialidades del sujeto no satisfechas y las exigencias del medio social cada vez más crecientes. Este dilema es consecuencia de la brecha que se ha originado entre la escuela y el medio social, en que cada uno "camina" por su lado sin vínculo concreto. No forman parte en la realidad de una complejidad.

Esto nos conduce a pensar que este dilema hay que abordarlo a partir de la integración de cinco elementos: los alumnos que aprenden y construyen los conocimientos, el docente y su forma de facilitar el aprendizaje en lo cognitivo, afectivo y valórico, la estructuración del curriculum, el gobierno del sistema educativo y el entorno escolar.

Por lo tanto las preguntas que se hagan deberán centrarse en el qué o cambio educativo; él por qué u objetivo que se persigue incluyendo los juicios de valor acerca de un acontecimiento; y él cómo o método que posibilita el cambio a partir de ideas y presunciones básicas que provoquen un modo constante de actuar; y él para qué.

\footnotetext{
${ }^{8}$ Kuhn T. ¿Qué son las revoluciones científicas? Ediciones Atlaya S.A. 1998. Barcelona. España
} 


\section{¿Y cómo se logra en el gestor este proceder?}

Kurt Lewin (1890 - 1947) figura clave en la teoría cognitivista del aprendizaje enfocó sus estudios no tanto al aprendizaje sino a la motivación y la percepción. Su concepto básico fue el de espacio vital, en que incluye todo lo que necesita saber una persona para comprender su conducta concreta en un ambiente psicológico específico en un tiempo dado. Estas teorías enfatizan en lo cognitivo: sensación, percepción, imaginación, recuerdo, pensamiento.

En la gestión del aprendizaje teniendo como base la integración de un modelo contextual-desarrollador con el enfoque de la complejidad, el aprendizaje resulta importante de tener en cuenta, porque el alumno aprende por medio de la generalización, diferenciación y la reestructuración de su personalidad y su ambiente psicológico. Así él logra adquirir nuevos significados y, de esta forma, cambian sus motivos, la preferencia por un grupo y las perspectivas del tiempo. De esta manera el sujeto obtiene mayor control de sí mismo y el mundo que le rodea.

\section{¿Qué gestión del aprendizaje debemos realizar pues?}

La concepción del aprendizaje como un proceso de construcción del propio alumno en un contexto dado propicia que el proceso parta de una situación como se presenta en la realidad, un problema convertido en necesidad, y el problema debe ayudar a realizar preguntas científicas, inferencias e hipótesis, a extraer reglas, principios y regularidades, a modular las emociones y sentimientos, a fomentar los valores que conducen a elevar el nivel cognitivo a partir de la tarea.

Como expresamos anteriormente, la tarea juega un papel significativo e importante en la gestión del pensamiento y puede ser solucionada a partir del conocimiento empíricamente construido por el sujeto, que se ocupa de las diferencias y semejanzas entre los fenómenos fundamentalmente dada por la observación y comparación, y puede ser resuelta también, por medio del conocimiento teórico, que reposa sobre un sistema de fenómenos no aislados, se construye por medio de métodos indagatorios en la resolución de las contradicciones en un área problemática central, desarrolla ideas sobre los orígenes, relaciones y dinámica de los fenómenos y, se comunica por medio de modelos. Se trata pues de facilitar acciones por el docente para que los alumnos hagan converger lo empírico y lo teórico en la solución de las tareas de manera integrada.

La importancia que tiene esto, es que va creando en el alumno una lógica entre conocimientos y comportamientos, entre lo teórico y lo procedimental, para que estos últimos se manifiesten a partir del cambio en el significado de la experiencia en lo social. Entonces: 


\title{
9. ¿Qué vamos a gestionar en la clase?
}

\author{
"Ya no se puede admitir que el profesor continúe siendo el sabio por \\ profesión frente al joven ignorante por definición, el profesor informador y el \\ alumno oyente tendrán que ser reemplazados por el profesor \\ animador y por el alumno investigador" \\ Correo de la UNESCO
}

Cuando un docente se hace cargo de un grado o curso, lo asaltan un sin número de criterios y si es recién contratado sea profesional o no, más. Lo primero que se encuentra es un curriculum cargado de contenidos que lo sumerge en la siguiente pregunta, ¿cómo voy a cumplir con todo esto? Si a esto se le añade que los alumnos en la sala de clases son numerosos, termina pensando cómo trasmitir el conocimiento para que el estudiante lo asimile de la forma más fácil.

Varios autores entre los que se encuentran Zubiria De (1994 y 1998), Blanco (1999), Silvestre y Zilberstein (2000), Olmedo (2000), Soubal (2003) han planteado las limitaciones que aun siguen registrándose en la praxis del docente y como consecuencia en el aprendizaje de los estudiantes:

- Insuficiente análisis e interpretación.

- Insuficiente búsqueda de información

- Pobre determinación de relaciones entre las cosas.

- Tendencias a la ejecución de forma reproductiva.

- Pocos procedimientos para aprender a aprender.

- Insuficiente desarrollo de la generalización y la reflexión.

- Deficiente comunicación.

- Deficientes habilidades para planificar el estudio y realización de tareas escolares.

La tendencia de los estudiantes es a la reproducción del conocimiento y no a su construcción, por lo que las propias limitaciones en el desarrollo de los estudiantes están centradas en el sistema educacional y en los docentes que no gestionan el aprendizaje eficazmente en correspondencia con las tendencias modernas.

"Ya no se puede admitir que el profesor continúe siendo el sabio por profesión frente al joven ignorante por definición, el profesor informador y el alumno oyente tendrán que ser reemplazados por el profesor animador y por el alumno investigador"9

La escuela debería ser un lugar distinto en que la gestión del aprendizaje propendiera

\footnotetext{
${ }^{9}$ Correo de la UNESCO. Una educación para el siglo XXI. Aprender a aprender ( pág.8) citado por Ziberstein y Portela en Una concepción desarrolladora de la motivación y el aprendizaje de las ciencias. La Habana. 2002
} 
al aprendizaje significativo, un lugar en que los alumnos se descongestionen de la polución mental, mejor aún, en donde se preparen con recursos valiosos, integralmente, para hacer frente a los niveles de polución mental que encontrarán a lo largo de su vida. Es por ello que ha cobrado fuerza en la formación, un concepto que integralmente revela las distintas facetas en el que el humano debe estar preparado, que propicie comportamientos para enfrentar los retos que le depara el proceso de la vida en niveles cualitativamente superiores. Este concepto es el de competencias, que aunque surgió como una necesidad en el plano de la actividad productiva, no se descarta por su amplitud que pueda ser utilizado en la formación general.

Este término es visto como la integración de conocimientos, habilidades, destrezas, actitudes, emociones y sentimientos, así como valores, y se debe empezar a tratar desde los primeros grados hasta que después puedan ser más específicos y relacionados con la labor que desarrollará el futuro profesional.

Es importante dominar que desde los primeros grados hay competencias genéricas de desarrollo personal que son necesarias potenciar y apoyar en su configuración, porque resulta ser la base para los comportamientos de los propios estudiantes y el futuro profesional. Estas son: la comprensión, la comunicación, la búsqueda de la información, la solución de problemas y la utilización del tiempo

Por ello la clase debe cambiar; que sirva para descubrir las potencialidades humanas y desencadenarlas, pasar de la enseñanza de teorías a la enseñanza del aprendizaje que es la verdadera identidad de la educación. Es tan cuantiosa la información que hay, que se requiere que el alumno sepa los fundamentos de los acontecimientos, y no tantas teorías que requerirían de una escuela interminable. Lo que si no cabe duda es que, se debe potenciar la formación de la escuela en la vida y eso necesitará humanos que estén preparados para aprender por sí solos en el contexto social. La respuesta a esto es que el hombre ante todo debe entender más su espacio vital, para comprenderse más, saber hasta dónde puede llegar, y cuáles son sus limitaciones para así al final poder hacer libremente.

Aunque hay docentes brillantes que gestionan el proceso de forma intuitiva y empíricamente, pensamos que el nuevo rol lo llevará a re-conceptualizar y clarificar sus prácticas educativas en una dimensión que apunte a la integración de lo científico con lo empírico. Las prácticas educativas deberán cambiar de clases modelos a los modelos de clases.

¿Y qué modelo de clases es el que debe primar en la gestión del aprendizaje de los gestores educacionales para contribuir al cambio en el significado de la experiencia en el quehacer educacional de la gestión escolar?

En correspondencia con la necesidad de retomar la verdadera identidad de la 
educación y darle direccionalidad a la gestión del aprendizaje, con el rol que le corresponde jugar al docente como mediador en la clase, es necesario adoptar un modelo didáctico que sirva de sostén, basado en el concepto de desarrollo humano en su acepción amplia, la relevancia del proceder investigativo y su marco teórico, así como la metodología correspondiente.

\section{0. ¿Qué es un modelo didáctico como sostén de la gestión del aprendizaje?}

La gestión del aprendizaje por el profesor en la sala de clases sé deriva de una forma de interpretar la realidad educativa en su contexto, vinculado con un marco conceptual psicopedagógico y gerencial, basados en principios, conformando ambos el marco teórico por el que debe actuar.

El modelo didáctico a utilizar debe estar centrado en el proceder investigativo como eje vertebral, ya que es la manera en que se puede abordar la diversidad de diferencias individuales en los alumnos. Es una complejidad que requiere la utilización de acciones dirigidas al abordaje de la complejidad a través del dilema que se le presenta al estudiante en una situación problemática y que marca la direccionalidad de la gestión en el sentido de movilizar las estructuras cognitivas del alumno, la dinámica grupal, lo emocional y los valores que se ponen de manifiesto en la solución del problema planteado. Esto implica evidentemente que los marcos curriculares sean flexibles para poderlos ir adaptando, puesto que lo óptimo sería que el currículum escolar tuviera como eje vertebral el principio del proceder investigativo. Por ello no debe realizarse un modelo curricular si el modelo pedagógico y su correspondiente modelo didáctico no están definido.

Es evidente que en este eje vertebrador los restantes componentes didácticos como el contenido, los objetivos, los métodos, las formas de organización del proceso docenteeducativo, los medios de apoyo y la evaluación deberán estar relacionados sistémicamente, no de forma analítica, que por cierto en la práctica se manifiestan de esta última forma. Es justo mencionar que si se desarrollan los enfoques por competencias aparecerá un nuevo componente didáctico que ya muchos autores lo consideran que es el problema

La integración en el orden metodológico, el enfoque de la complejidad, la planificación, los aspectos psicológicos de los sujetos y el currículo nos da un marco global, una filosofía para abordar el aprendizaje desde el punto de vista de la gestión docente.

Basados precisamente en el modelo didáctico con el eje vertebrador en el proceder investigativo, se podrán trabajar modelos de clase que propendan a la estimulación el pensamiento así como al aprendizaje por parte de los estudiantes. 


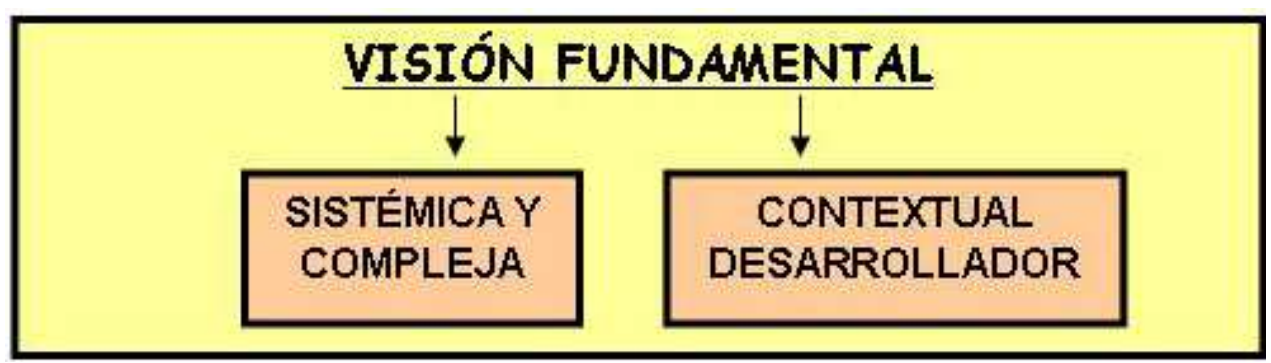

Las acciones necesarias para abordar la gestión del aprendizaje basadas en éste modelo didáctico, deberán partir de la activación del pensamiento, que significa poner a los alumnos en plena disposición para el aprendizaje. Las acciones son las siguientes:

- La facilitación de actitudes y percepciones positivas sobre el aprendizaje incluido el clima psicológico propicio.

- El razonamiento para la adquisición e integración del conocimiento. Todo conocimiento nuevo debe integrarse a los que posee el alumno previamente.

- Destrezas del pensamiento involucradas en la extensión y refinamiento del conocimiento entre las que se encuentran: comparación, clasificación, inducción, deducción, análisis de errores, apoyo, abstracción, análisis de valores y el desarrollo de síntesis.

- Uso significativo del conocimiento que se logra a partir de la resolución de tareas a largo plazo, tareas multidimensionales, tareas dirigidas al estudiante, resolución de problemas, adopción de decisiones, invención e indagación experimental.

- Hábitos mentales productivos que son consecuencia de actividades tales como: planeación, pensamiento crítico y pensamiento creativo.

Por supuesto que el desarrollo del pensamiento en los estudiantes posibilitará ir configurando a lo largo de sus estudios un pensamiento de orden superior del tipo criticreativo, que estará muy vinculado con los comportamientos que él desarrolle después de su egreso. A continuación la tabla 1 muestra la relación del pensamiento con los tipos de conductas que es necesario potenciar en el proceso enseñanza-aprendizaje para garantizar ese tipo de reflexión en el estudiante.

Es evidente entonces que, después de involucrar a los alumnos en la activación de su pensamiento, el profesor deberá pensar en las estructuras de acciones o métodos que utilizará para lograr llegar al cambio deseado en la gestión docente de su clase. La pregunta lógica que se hará será la siguiente:

¿Qué métodos utilizaré en la gestión del aprendizaje? 


\section{Tabla 1}

\begin{tabular}{|c|c|c|c|c|}
\hline $\begin{array}{c}\text { PENSAMIENTO } \\
\text { ETAPAS }\end{array}$ & $\begin{array}{l}\text { PENSAMIENTO } \\
\text { CRITICO } \\
\text { DESTREZAS }\end{array}$ & CONDUCTAS & $\begin{array}{l}\text { PENSAMIENTO } \\
\text { CREATIVO. } \\
\text { DESTREZAS }\end{array}$ & CONDUCTAS \\
\hline Senso-percepción & interpretación & $\begin{array}{l}\text { categorización } \\
\text { decodificación } \\
\text { y clasificación de } \\
\text { significados }\end{array}$ & apertura mental & $\begin{array}{l}\text { escuchar y estar } \\
\text { abierto a que otros } \\
\text { discrepen de tu } \\
\text { posición }\end{array}$ \\
\hline Análisis & análisis & $\begin{array}{l}\text { examinar ideas } \\
\text { identificar y analizar } \\
\text { argumentos }\end{array}$ & indagación & $\begin{array}{l}\text { buscar } \\
\text { Investigar. } \\
\text { formular problemas }\end{array}$ \\
\hline Síntesis & evaluación & $\begin{array}{l}\text { valorar enunciados y } \\
\text { argumentos }\end{array}$ & razonamiento & $\begin{array}{l}\text { relacionar partes, } \\
\text { relacionar partes con } \\
\text { el todo y viceversa }\end{array}$ \\
\hline comparación & inferencia & $\begin{array}{l}\text { examinar evidencias, } \\
\text { contrastar hechos y } \\
\text { datos conjeturar y } \\
\text { deducir }\end{array}$ & razonamiento & $\begin{array}{l}\text { Relacionar, contrastar } \\
\text { hechos y datos, crear } \\
\text { modelos }\end{array}$ \\
\hline abstracción & explicación & $\begin{array}{l}\text { fundamentar } \\
\text { justificar } \\
\text { enunciar resultados }\end{array}$ & Apertura mental & $\begin{array}{l}\text { Crear modelos, } \\
\text { conceptos, juicios, } \\
\text { ideas acerca de algo }\end{array}$ \\
\hline generalización & autorregulación & $\begin{array}{l}\text { Auto examinarse } \\
\text { Auto corregirse }\end{array}$ & razonamiento & $\begin{array}{l}\text { Buscar las } \\
\text { regularidades de algo }\end{array}$ \\
\hline
\end{tabular}

Durante muchos años los docentes se han preguntado:

¿Para qué enseñamos en las diferentes edades? ¿Qué ciencia enseñamos y cómo la enseñamos?

Estas viejas preguntas requieren de respuestas innovadoras y creativas, que sitúen a la educación al igual de los procesos científicos que vive la humanidad en cualquier campo del proceder humano y que se elimine el peso enciclopedista y conceptual basada en la lógica de los contenidos.

Recordemos que educación proviene de la palabra educare, que significa conducir, guiar, orientar y educere, hacer salir, dar a luz. Por lo tanto es la actividad mediante la cual los grupos humanos promueven su desarrollo personal para actuar en la sociedad a lo largo de su vida.

Los modos de actuar de los docentes en la gestión del aprendizaje deben ir más allá de simples formas centradas en los contenidos curriculares y requiere de la actualización pedagógica constante con vistas a ampliar su bagaje cultural y tener mayores posibilidades de métodos que se opongan a los utilizados en los modelos clásicos o los utilizados por aquellos docentes cuyas prácticas son puramente intuitivas. $\mathrm{Se}$ trata de 
conducir o guiar el aprendizaje en las clases novedosamente, con el objetivo como referencia en relación con los contenidos, a partir de lo que cada situación demanda como expresión de una necesidad con vistas a satisfacerla.

En primer lugar, planteamos en las partes precedentes de esta obra, que el método a aplicar con carácter global debe ser el del tránsito en cualquiera de las actividades que se realicen del todo en un acontecimiento a las partes para después regresar al todo integrado. La utilización de este enfoque como estructura del pensar en general, va acompañado también de otros métodos específicos en correspondencia con los conocimientos que se quieran construir y que los docentes facilitarán en su papel de mediador.

Estos otros métodos específicos que pudieran ser utilizados son: la modelación, la simulación, el análisis sistémico, el dialógico y el método de elaboración de proyectos, entre otros. Todos ellos integrados en el proceder investigativo como modelo didáctico conducen a que el estudiante trabaje independientemente o en equipo en las tareas con su sello característico, abandonando las prácticas reproductivas en el aprendizaje y propiciando la convergencia entre el pensar y el hacer en función de lograr aprendizajes significativos.

El método de la modelación resulta de mucha utilidad, porque el mundo es una representación en que se resaltan aquellas interdependencias que posibilita penetrar en lo complejo. El profesor al facilitar el aprendizaje de la modelación, permite reunir los principales componentes de un sistema para establecer interrogantes e hipótesis sobre su comportamiento global. Toda imagen mental es un modelo, borroso e incompleto, pero que sirve de apoyo a decisiones ulteriores. Análisis simples y medianamente complejos se pueden realizar por medio de este método. Su utilización posibilita la convergencia de las dimensiones de la gestión en sus tres aristas cognitivas, afectivas y valóricas.

La simulación estudia los sistemas que requieren mayor complejidad dado que permite ver el funcionamiento de un acontecimiento en sus tres dimensiones. $\mathrm{O}$ sea, todas las variables al unísono que es lo que sucede en la realidad. Tal es el caso de los simuladores de vuelo, simuladores para aprender a manejar o los ordenadores modernos.

El método de análisis de sistemas ayuda a los alumnos a delimitar el sistema a modelar, en identificar los componentes y las interacciones entre ellos, de manera tal que, puedan priorizar y organizar en niveles jerárquicos. Después se resaltan las variables a tener en cuenta.

Estos dos últimos métodos descritos ponen de manifiesto también, la utilización de las dimensiones de la gestión con sus aristas cognitivas, afectivas y valóricas en el proceso de construcción de conocimientos y aprendizajes. 
El método dialógico permite al docente conducir la clase y facilitar que los alumnos muevan sus mentes hacia delante y hacia atrás al ritmo que el profesor estime y que los alumnos puedan enfrentar. Esta estructura de acciones en la clase busca el acercamiento paulatino al conocimiento que se construye a partir de lo relacional y racional. Pone al alumno en situaciones en que emite juicios críticos acerca de algo y posibilita que se trabajen las dimensiones de la gestión escolar con sus tres aristas. Posibilita además, poner al alumno en el centro de los acontecimientos que se analizarán.

El método de la realización de proyectos pasa en primer lugar por dominar lo que es un proyecto. Este no es más que la esencia de un proceso que se desea modelar a partir de objetivos definidos. En su diseño se expresan las ideas y se fundamenta científicamente lo que ulteriormente se va a acometer. A partir de este método el profesor posibilita que el alumno genere y estructure ideas, haga cosas, cree, aporte teóricamente en su fundamentación, busque costos y defina el impacto que tendrá el proyecto. El estudiante se prepara para defender las concepciones adoptadas en su realización. El profesor desde su posición de facilitador ayuda en la planificación, apoya en su realización y supervisa el proceso de construcción de los proyectos. Los proyectos pueden ser trabajados individualmente o por equipos y hay que darle tiempo para su construcción. A partir de la utilización de esta metodología se garantiza integralmente el abordaje de las aristas cognitivas, afectivas y valóricas.

Estos métodos que hemos descritos y otros que pudieran utilizarse y que no se describen en esta obra unido a técnicas como la UVE y la elaboración de mapas conceptuales que propician la construcción de conocimientos y aprendizajes significativos, pueden contribuir a que los estudiantes desarrollen sus capacidades intelectuales y las que se requieran para vivir en las sociedades contemporáneas a partir del aprendizaje de la autoreferencia, la creatividad, la autoevaluación, la reflexión crítica y los juicios morales. Desarrollan distintas inteligencias al decir de Howard Gardner, y los gestores pueden detectar los comportamientos del alumnado en las distintas actividades con mayor apego a uno u otro.

No cabe la menor duda que toda esta tecnología intelectual que pudieran utilizar los gestores en su gestión del aprendizaje, pone al alumno en el centro del proceso docente educativo y da una opción innovadora en la aventura de facilitar el enfrentamiento a los retos que no da la gestión del aprendizaje basado en el modelo didáctico del proceder investigativo.

\section{Conclusiones}

El desarrollo de la educación a lo largo de estos años, en particular en la segunda mitad del siglo XX y el inicio del siglo XXI ha provocado cambios profundos en la epistemología pedagógica y sobre todo en temas tan importante y complejo como el aprendizaje, que va desde el empirismo ingenuo hasta teorías cognitivas centradas en el sujeto y otras con marcado énfasis en el desarrollo histórico-cultural. 
Hoy vamos al desarrollo de un aprendizaje más cualitativo para los escolares, por lo que se requiere que los docentes se superen, actúen sobre la base de modelos teóricos para hacer la praxis escolar más científica, comprendan los fenómenos que están implícitos en el proceso del aprendizaje y diseñe estrategias para su puesta en práctica.. Se requiere que los sujetos dominen su espacio vital para que puedan comprender lo que sucede en la sala de clases con los humanos que comparten el proceso de construcción del conocimiento, su génesis histórica y que los cambios no atropellen el proceso lógico natural que se requiere en construcciones de este tipo.

Todos los gestores educacionales estamos comprometidos con la constante renovación del saber, el saber hacer y el convivir, a comprender al humano y qué es lo que lo motiva a actuar en un momento determinado. Esa es la dirección en que se debe trabajar la gestión que hagamos en pos del aprendizaje, para que los alumnos se percaten del sentido que tiene aprender y la ponderación para la vida de ésta cualidad del ser humano. 


\section{Bibliografía}

Arellano d Lancino N Ms. 2003 La realidad exige una nueva educación. www.monografías.com Enero

Lipman M. 2003 La filosofía en el aula. Ediciones de la Torre. Re - editado por el Liceo Católico de Atacama (nivel Básico). Copiapó. Chile.

Delgado C. 1999 Artículo publicado en la revista de Filosofía de la Ciencia. Vol. 1, Colombia pág. 28

Gómez T. 2003 Tesina en opción al Diplomado Enfoque Contemporáneo del Proceso Docente-Educativo. Copiapó. Chile

Kuhn T. 1998 ¿Qué son las revoluciones científicas? Ediciones Atlaya S.A. Barcelona.

López A. (2003) El proceso Enseñanza - Aprendizaje de la Educación Física. Editorial Deportes. La Habana. Cuba

Minunjin A Dra. 1994 Una investigación pedagógica transferida a la práctica en las escuelas cubanas. Selección de Artículos.

Novak y Gowin D.B. 1999 Aprendiendo a aprender. Ediciones Martínez Roca(2a edición) Barcelona. España

Rosnay DJ. 1975 Hacia una visión global. Editorial AC. Madrid. España.

Todos O. 1990 Metodología para la organización con enfoque de sistemas. Revista cubana de Educación Superior. La Habana.

Soubal Caballero S. Dr. 1993 Algunas regularidades pedagógicas en el resumen del curso escolar 92-93 MM. La Habana.

Soubal Caballero S. Dr. 2004 Algunas consideraciones sobre la gestión del aprendizaje y el modelo didáctico del proceder investigativo. Diplomado dictado en INACAP. La Serena. Chile 